\title{
Nonclinical Time for Family Medicine Residency Faculty: National Survey Results
}

Jennie B. Jarrett, PharmD, MMedEd | Simon Griesbach, MD | Mary Theobald, MBA | Jeffrey D. Tiemstra, MD | David Lick, MD

PRiMER. 2021;5:45.

Published: 12/1/2021 | DOI: 10.22454/PRiMER.2021.338005

\section{Abstract}

Introduction: Recent changes to the Accreditation Council for Graduate Medical Education (ACGME) requirements eliminated minimum standards for protected nonclinical time for core faculty. Faculty perform many nonclinical tasks to maintain family medicine residencies. The objective of this study is to describe the landscape of nonclinical time for family medicine residency faculty.

Methods: Program directors at ACGME-accredited family medicine residencies were electronically surveyed in August 2019 to describe nonclinical time of their faculty. Survey information requested included program demographics, the amount of nonclinical time allocated, and the estimated amount of nonclinical time spent per year completing their faculty duties.

Results: A total 156 of 635 program directors (24.6\%) returned the survey and $58(9.1 \%)$ completed the entire survey for analysis inclusion. An average of 3,394 hours per year, per program were estimated to be spent on nonclinical activities. The greatest amount of time was spent on program administration (39\%) and the least amount of time on curriculum development (5\%). There was variation in the use of nonphysician faculty to complete these tasks. Allocated faculty time was comparable to estimated time spent performing nonclinical tasks. On average, a 24-resident program devoted 1.9 full-time equivalent faculty salary support to complete nonclinical activities.

Conclusions: Family medicine residency faculty spend significant time completing nonclinical tasks required to meet ACGME requirements and need the protected time to complete these necessary tasks. Direct data on the use of faculty nonclinical time is needed to more accurately define its use.

\section{Introduction}

Prior to 2019, the Accreditation Council for Graduate Medical Education (ACGME) family medicine residency program requirements specified minimum protected time for program directors (PDs) and faculty to perform nonclinical work. Faculty members perform nonclinical activities such as developing residency curricula, evaluating residency applicants, assembling and delivering didactic lectures, evaluating and providing feedback, and performing independent scholarly activity, which is important for an adequate learning experience of residents. ${ }^{1,2}$ Effective July 2019 , the common program requirements include a requirement for minimum protected nonclinical time for PDs and associate PDs, but removed the option to set protected time 
requirements for core faculty. ${ }^{3}$ This was enacted to standardize nonclinical time, which varied widely between specialties. ${ }^{4,5}$

The actual amount of time spent by family medicine PDs and faculty for nonclinical tasks has not been studied. Data from the ACGME indicate family medicine PDs allocated approximately $60 \%$ and core faculty allocated approximately $27 \%$ of their time to these activities. ${ }^{6}$ However, there is limited national data regarding actual time spent by family medicine faculty in nonclinical activities.

Attitudes of too much patient care and resident supervision are associated with a higher desire to leave academic medicine for family medicine faculty. ${ }^{7}$ Faculty members identified workload/administrative burden/competing priorities as their biggest work-related challenge, noting that higher clinical demands compromised their nonclinical responsibilities. ${ }^{8}$ This challenge is predicted to worsen with the new ACGME guidance.

The objective of this study was to estimate the current amount of time faculty spend performing nonclinical duties, how that compared to allocated nonclinical time, and how the time allocated or spent differed based on faculty demographics or other program characteristics.

\section{Methods}

\section{Survey Development}

The STFM Graduate Medical Education Committee developed a 13-item survey to evaluate the amount of time that family medicine residency program faculty spend performing various nonclinical functions required for ACGME accreditation. The STFM Research Committee and leaders of the Association of Family Medicine Residency Directors (AFMRD) reviewed survey questions. Based on feedback, survey questions were modified to increase clarity and face validity. The survey included demographic questions, questions about time spent on specific activities related to program administration, and a free-text chart asking for the percent full-time equivalent (FTE) each faculty member spent on clinical care, resident supervision, and nonclinical time. The full survey is available on the STFM Resource Library. ${ }^{9}$

\section{Survey Collection}

The survey sampling frame was PDs from ACGME-accredited family medicine residency programs in the United States listed in the 2018-2019 ACGME directory of residency programs. PDs were targeted as the group most able to accurately estimate time spent and allocated for all faculty within a program. The first author (J.B.J.) delivered the survey to this group via an internet-based survey instrument (Qualtrics) in August 2019. Nonresponders received three reminder emails at weekly intervals to encourage participation. The survey remained open and available for participation for 5 weeks.

\section{Data Analysis}

We only included completed surveys in the analysis. We used descriptive statistics for continuous variables to summarize the data. The Institutional Review Board at the University of Illinois at Chicago approved this study.

\section{Results}

\section{Participants}

We identified a total of 635 family medicine residency PD emails. Of 156 PDs that returned their survey (24.6\% response rate) only 58 were completed in their entirety and included in the analysis ( $9.1 \%$ completion rate). Programs averaged a total of 22 residents and 11 faculty members. Demographics are found in Table 1. 


\section{Faculty Time Allocation}

PDs reported on a total of 600 faculty, including physicians, behavioral health specialists, pharmacists, and others. For all types of faculty, the average FTE allocation for clinical time without a resident, clinical time with a resident, and nonclinical time was 0.29 ( \pm 0.21 SD), 0.33 ( \pm 0.20 SD), and $0.26( \pm 0.20$ SD), respectively. Physician faculty were allocated more clinical time with residents compared to other groups, while behavioral health faculty were allocated more nonclinical time (Table 2). Faculty time allocation based on profession and program size are shown in Table 3.

\section{Faculty Use of Nonclinical Time}

The average total time estimated spent on nonclinical faculty activities was 3,394 hours per program, per year, or 65 hours per program per week. The average total amount of time spent per faculty per year was 309 hours. The largest amount of time was spent on administration of the program (1,327 hours, 39\%), the least amount of time was spent on curriculum development (162 hours, $5 \%$ ). Average faculty time spent on specific nonclinical activities are shown in Table 4.

\section{Discussion}

This is the first study to characterize nonclinical time for family medicine residency faculty nationwide. Based on the total number of hours spent per program per year (3,394 hours), meeting ACGME requirements necessitates a substantial nonclinical time commitment. PDs estimate that faculty spend approximately 156.5 nonclinical hours per year per resident in the program. This equates to a 1.9 faculty FTE of nonclinical time for a 24-resident program based on a 40-hour workweek and 46 work weeks/year. Although physicians composed the majority of faculty reported on (86\%), the data also demonstrate the contributions of pharmacy, behavioral health, and other disciplines to nonclinical residency duties in addition to their contributions to residents' clinical training and patient care.

Reported estimates of hours spent on nonclinical activities totaled less than allocated time (.26 versus .17 FTE). The wide variation in individuals' reported times, whether allocated or estimated actual time spent, demonstrate that programs find different and unique ways to distribute the workload among faculty. The breakdown of nonclinical activities challenges us to consider the value we place on certain activities. Administrative activities occupied the largest use of time at $39 \%$, while curriculum development, scholarship, and faculty development time were all estimated at only 5\%-6\% each. The lesser time utilized across curriculum development, scholarship, and faculty development are of particular interest with their critical nature for improvement of residency education and the family medicine specialty as a whole, and these areas are often perceived as taking tremendous amounts of faculty nonclinical time. It may be prudent for residency programs to determine alternative mechanisms for administrative tasks or offsetting of clinical time in order to focus nonclinical time toward higher-value tasks that directly impact the quality and sustainability of the program. ${ }^{10}$

This study has several limitations. The sample size was only $9.1 \%$ of all US-based family medicine residencies, although the broad geographic, population density, and program size distributions suggest a representative sample. The survey required significant amounts of detail and time that likely limited the PDs' ability to complete accurately, particularly in programs with many faculty, causing this low response rate. Also, PDs' estimates of their faculty members' use of time is an administrative impression of their work and may undercount time, including time spent outside of normal working hours. Finally, PDs' knowledge of actual time allocations for their faculty may have altered their reported estimates of their faculty members' actual time spent. This could partly explain the similarity between estimated hours spent and time allocation in our study. Future research examining faculty time directly may lead to more accurate data. 
This study provides the first description of nonphysician faculty time allocation and incorporation of nonphysician faculty members' effort into time spent within family medicine residencies. Given limited literature available on nonphysician faculty members, this may be interesting to those seeking to understand the substantive contributions of nonphysician faculty members. It does not tell us if or how faculty time use correlates to program quality or performance, any metric of patient care, or graduate quality over time.

\section{Conclusions}

Family medicine residency faculty spend significant time completing nonclinical tasks and need the protected time to complete these necessary tasks. These data may serve as a discussion starter for appropriate allocation of residency faculty time to accomplish their educational mission and accreditation. Further research should include prospective time studies, and also consider whether the amount and distribution of faculty nonclinical time correlates with accepted measures of program quality such as board examination performance, graduation rates, or clinical quality of care.

\section{Tables and Figures}

Table 1: Demographics of Family Medicine Residency Programs ( $\mathrm{n}=58$ Programs)

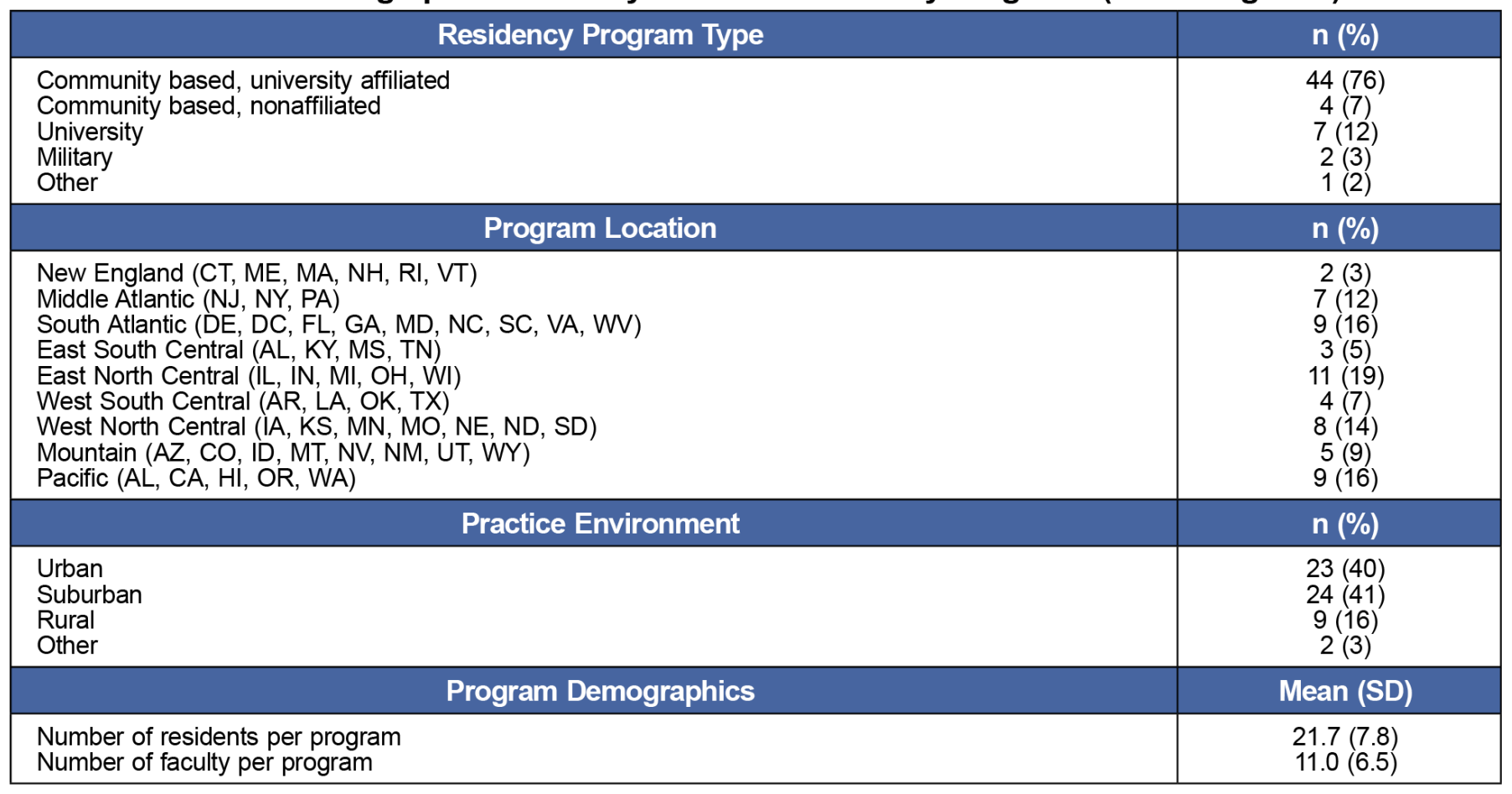


Table 2: Faculty Time Allocation in Full-Time Equivalents ( $n=58$ Programs)

\begin{tabular}{|l|c|c|c|}
\hline & \multicolumn{3}{|c|}{ FTE Allocation, Average (SD) } \\
\hline \multicolumn{1}{|c|}{ Profession by Degree } & Clinical, No Resident & Clinical, With Resident & Nonclinical \\
\hline MD/DO $(n=516)$ & $0.29(0.20)$ & $0.35(0.20)$ & $0.25(0.18)$ \\
\hline PharmD $(n=20)$ & $0.22(0.22)$ & $0.21(0.20)$ & $0.23(0.27)$ \\
\hline BH/PsyD ( $n=45)$ & $0.31(0.23)$ & $0.23(0.19)$ & $0.38(0.26)$ \\
\hline $\begin{array}{l}\text { Other (MSW, JD, PhD, } \\
\text { unknown) (n=19) }\end{array}$ & $0.19(0.23)$ & $0.25(0.22)$ & $0.37(0.30)$ \\
\hline All Faculty (n=600) & $\mathbf{0 . 2 9 ( 0 . 2 1 )}$ & $\mathbf{0 . 3 3 ( 0 . 2 0 )}$ & $\mathbf{0 . 2 6 ( 0 . 2 0 )}$ \\
\hline
\end{tabular}

Abbreviations: FTE, full-time equivalent; $\mathrm{BH}$, behavioral health.

Table 3: Faculty Time Allocation in FTEs, Based on Profession and Program Size (n=58 Programs)

\begin{tabular}{|c|c|c|c|c|c|c|c|}
\hline \multirow[b]{2}{*}{ Profession } & \multirow[b]{2}{*}{$\begin{array}{l}\text { Type of Time } \\
\text { Utilization }\end{array}$} & \multicolumn{6}{|c|}{ Residents, (Number of Programs) } \\
\hline & & $<12(4)$ & $\begin{array}{c}12 \text { to } \\
17(11)\end{array}$ & $\begin{array}{c}18 \text { to } \\
23(13)\end{array}$ & $\begin{array}{l}24 \text { to } \\
29(21)\end{array}$ & $\begin{array}{l}30 \text { to } \\
35 \text { (6) }\end{array}$ & $36+(3)$ \\
\hline \multirow{2}{*}{$\begin{array}{l}\text { MD/DO } \\
(n=516)\end{array}$} & Clinical, resident & 0.38 & 0.29 & 0.34 & 0.42 & 0.33 & 0.27 \\
\hline & Nonclinical & 0.21 & 0.23 & 0.22 & 0.26 & 0.28 & 0.20 \\
\hline \multirow{2}{*}{$\begin{array}{l}\text { PharmD } \\
(n=20)\end{array}$} & Clinical, resident & 0 & 0.10 & 0.28 & 0.21 & 0.24 & 0.00 \\
\hline & Nonclinical & 0.5 & 0.10 & 0.20 & 0.33 & 0.10 & 0.00 \\
\hline \multirow{2}{*}{$\begin{array}{l}\mathrm{BH} / \mathrm{PsyD} \\
(\mathrm{n}=45)\end{array}$} & Clinical, no resident & 0.4 & 0.44 & 0.18 & 0.36 & 0.13 & 0.60 \\
\hline & Clinical, resident & 0.4 & 0.16 & 0.28 & 0.28 & 0.18 & 0.00 \\
\hline Other $(n=19)$ & Nonclinical & 0 & 0.20 & 0.43 & 0.25 & 0.00 & 0.50 \\
\hline
\end{tabular}

Table 4: Average Faculty Time Spent on Specific Nonclinical Activities ( $n=58$ Programs)

\begin{tabular}{|l|c|c|c|c|c|}
\hline \multirow{2}{*}{ Use } & \multicolumn{2}{|c|}{$\begin{array}{c}\text { Hours/Year } \\
\text { per Program* (SD) }\end{array}$} & $\begin{array}{c}\text { Hours/Month } \\
\text { per Program* (SD) }\end{array}$ & $\begin{array}{c}\text { Hours/Year } \\
\text { per } \\
\text { Resident }\end{array}$ & $\begin{array}{c}\text { Hours/Year } \\
\text { per Faculty }\end{array}$ \\
per Faculty
\end{tabular}

Abbreviation: FTE, full-time equivalent

* Entries with an SD reported represent survey data; other entries were calculated from survey data. 


\section{Acknowledgments}

The authors acknowledge the 2019-2020 STFM Graduate Medical Education Committee for their input throughout this work.

\section{Corresponding Author}

Jennie B. Jarrett, PharmD, MMedEd

833 S. Wood Street (MC886), Chicago, IL 60612. 312-996-1098.

Jarrett8@uic.edu

\section{Author Affiliations}

Jennie B. Jarrett, PharmD, MMedEd - Department of Pharmacy Practice, College of Pharmacy, University of Illinois at Chicago, Chicago, IL

Simon Griesbach, MD - Waukesha Family Medicine Residency at ProHealth Care, Waukesha, WI

Mary Theobald, MBA - Society of Teachers of Family Medicine, Leawood, KS

Jeffrey D. Tiemstra, MD - Advocate Aurora Health Care, Elkhorn, WI

David Lick, MD - William Beaumont Hospital, Family Medicine Residency, Troy, MI I Department of Family Medicine, Oakland University William Beaumont School of Medicine, Troy, MI

\section{References}

1. Westberg SM, Hlavenka LM, Moon JY, Pereira CR. Time study of pharmacy practice faculty in family medicine residency clinics. Curr Pharm Teach Learn. 2011;3(2):85-91. doi:10.1016/j.cptl.2011.01.006

2. Butler DJ, Brocato J, Yeazel M. Family medicine didactics revisited. Fam Med. 2017;49(10):778-784.

3. ACGME Common Program Requirements (2017 Update). Accreditation Council for Graduate Medical Education. Accessed August 5, 2018. https://www.acgme.org/Portals/0/PFAssets /ProgramRequirements/CPRs_2017-07-01.pdf

4. Accreditation Council for Graduate Medical Education. Specialty-specific references for DIOs: Expected Time for Faculty. Published online June 2018. Accessed November 17, 2021. https://www.acgme.org /Portals/0/PDFs/Specialty-specific\%20Requirement\%20Topics/DIO-Expected_Time_Faculty.pdf

5. Specialty-specific References for DIOs: Expected Time for Program Director. Accreditation Council for Graduate Medical Education. Published online July 2019. Accessed November 17, 2021. https://www.acgme.org/Portals/0/PDFs/Specialty-specific\%20Requirement\%20Topics/DIOExpected_Time_PD.pdf

6. Department of Applications and Data Analysis, Accreditation Council for Graduate Medical Education. Data Resource Book (2007-2019). Accreditation Council for Graduate Medical Education; 2019.

7. Pollart SM, Novielli KD, Brubaker L, et al. Time well spent: the association between time and effort allocation and intent to leave among clinical faculty. Acad Med. 2015;90(3):365-371. doi:10.1097/ACM.0000000000000458

8. Theobald M. STFM Advocates for Protected Nonclinical Time for Residency Faculty. Ann Fam Med. 2019;17(5):467-468. doi:10.1370/afm.2455

9. Jarrett J. Non-Clinical Faculty Time Survey from the STFM GME Committee. 2021. STFM Resource Library. Accessed November 17, 2021. https://resourcelibrary.stfm.org/viewdocument/non-clinicalfaculty-time-survey-fr?CommunityKey=2751b51d-483f-45e2-81 de-4faced0a290a\&tab=librarydocuments

10. Greenberger SM, Finnell JT, Chang BP, et al. Changes to the ACGME Common Program Requirements and their potential impact on emergency medicine core faculty protected time. Chan T, ed. AEM Educ Train. 2020;4(3):244-253. doi:10.1002/aet2.10421 
Copyright $(\subset 2021$ by the Society of Teachers of Family Medicine 